\title{
Localization of mouse hepatitis virus open reading frame 1A derived proteins
}

\author{
Weizhen Bi ${ }^{1,5}$, Josefina D Piñón ${ }^{2}$, Scott Hughes ${ }^{2,6}$, Pedro J Bonilla ${ }^{2,7}$, Kathryn V Holmes ${ }^{3}$, Susan R Weiss ${ }^{2}$ and \\ Julian L Leibowitz ${ }^{4}$ \\ ${ }^{1}$ Department of Pathology and Laboratory Medicine, University of Texas Health Sciences Center, Houston, Texas 77225; \\ ${ }^{2}$ Department of Microbiology, University of Pennsylvania, Philadelphia, Pennsylvania 19104-6076; ${ }^{3}$ Department \\ Microbiology, CB B-175, University of Colorado Health Science Center, Denver, Colorado 80262; ${ }^{4}$ Department of \\ Pathology and Laboratory Medicine, Texas A\&M Health Science Center, 208 Reynolds Building, College Station, Texas \\ 77843-1114, USA
}

\begin{abstract}
We have investigated the intracellular localization of proteolytic cleavage products encoded in the $5^{\prime}$ portion of mouse hepatitis virus (MHV) gene 1. Immunofluorescent labeling of cells with an antiserum which recognizes p28, the ORF1a N-terminal cleavage product, resulted in widespread somewhat granular cytoplasmic staining, indicating that this protein is widely distributed in the cytoplasm of MHV-infected, but not control uninfected cells. Immunofluorescent staining of infected cells with antisera which recognize the downstream polypeptides, p65, p240 and p290 labeled discrete vesicular perinuclear structures. Double immunofluorescent labeling of BHK cells expressing the MHV receptor $\left(\mathrm{BHK}_{\mathrm{MHVR} 1}\right)$ and infected with MHV-A59 with a Golgi-specific anti-mannosidase II monoclonal antibody and with antiserum recognizing each of these anti-MHV ORF1a polypeptides, showed that the p240 and p290 polypeptides were localized in discrete vesicular structures that overlapped the Golgi complex. Labeling with antibodies specific for p65 colocalized with the Golgi region, and showed staining of the perinuclear cytoplasm as well. Plasmids containing sequences contained in the first $6.75 \mathrm{~kb}$ of ORF1a have been expressed using the coupled vaccinia virus-T7 polymerase system. Immunofluorescent labeling of transfectants with the anti-ORF1a antisera showed patterns of antigen distribution similar to those observed in cells infected with MHV-A59. A deletion analysis with constructs containing only portions of the ORF1a sequence indicated that 303 amino acids containing the first papain-like protease domain (PLP-1) was sufficient to associate this protein with the Golgi.
\end{abstract}

Keywords: coronavirus; proteolytic protein processing; protein trafficking

\section{Introduction}

Coronaviruses are widespread in nature, infect many vertebrate hosts and are important veterinary pathogens (Siddell et al, 1983). The human coronaviruses are most commonly associated with respiratory infections although there have been several reports associating coronaviruses with multiple sclerosis (Tanaka et al, 1976; Burks et al, 1980; Murray et al, 1992; Talbot et al, 1993). The

Correspondence: JL Leibowitz

Present addresses: ${ }^{5}$ Division of Cardiology, Department of Medicine, University of Texas Health Sciences Center, Houston, Texas 77225; ${ }^{6}$ Department of Microbiology, Columbia University, New York, NY 10032; ${ }^{7}$ Division of Molecular Virology, Baylor College of Medicine, Houston, Texas 77030, USA

Received 24 February 1998; revised 4 August 1998; accepted 5 August 1998 murine coronavirus mouse hepatitis virus (MHV), causes a broad spectrum of diseases in mice, depending upon the virus used, the route of inoculation, and the age and strain of a mouse (Siddell et al, 1983). Two strains of MHV, MHVA59 and MHV JHM, produce an encephalomyelitis which extensively involves the white matter of the brain with demyelinating lesions of the spinal cord (Weiner, 1973; Lavi et al, 1984). Infection with these viruses provides a useful small animal model for multiple sclerosis.

MHV contains a single-stranded positive-sense RNA genome (Spaan et al, 1988). One of the initial steps in MHV replication is the translation of gene 1 from the input genomic RNA. Gene 1 encompasses approximately $21.8 \mathrm{~kb}$ at the $5^{\prime}$ end of the genome 
and contains two overlapping large open reading frames (Lee et al, 1989; Bonilla et al, 1994). The first open reading frame ORF1a encodes 4469 amino acids. The second open reading frame ORF1b, overlaps ORF1a for 75 nucleotides and is 2771 amino acids long. In vitro transcription and translation studies of gene 1 have indicated that these two ORFs are translated by a ribosomal frameshifting mechanism to yield a polyprotein of almost $800000 \mathrm{kDa}$ which is co-translationally cleaved (Breedenbeek et al, 1990). Analysis of the nucleotide sequence and the predicted amino acid sequence revealed that ORF1a contains several likely functional domains: two hydrophobic domains, three cysteine-rich domains, a picornavirus 3C-like protease domain, and two papain-like protease domains (Lee et al, 1989; Bonilla et al, 1994). The relative positions of these ORF1a domains are depicted schematically in Figure 1. ORF1b is predicted to encode domains required for the MHV RNA-dependent RNA polymerase activ-

\section{MHV ORF 1a}

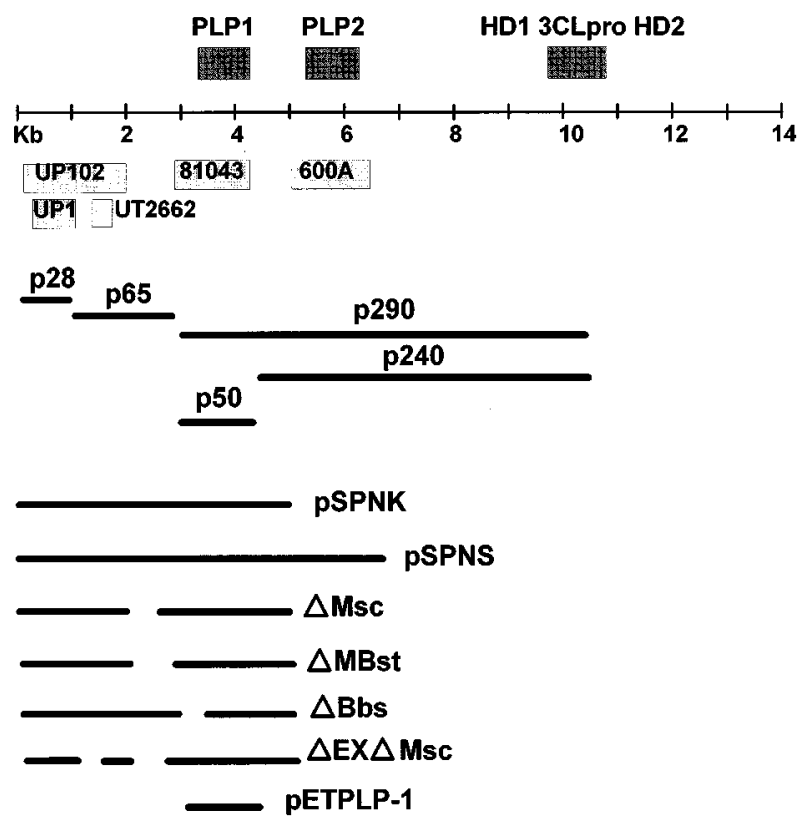

Figure 1 A schematic representation of the locations of predicted functional domains of MHV ORF1a. The positions of the two predicted papain-like protease domains, PLP1 and PLP2; the position of the predicted picornavirus 3C-like protease (3CLpro) domain and its two flanking hydrophobic domains, HD1 and HD2, are indicated by the labeled shaded boxes. The approximate locations of the ORF1a polypeptides used as immunogens and the names of the resulting antisera (UP1, UP102, 81043, 600A are shown in boxes below the kilobase scale. The approximate lengths and map positions of the MHV ORF1a inserts in the plasmids pSPNK, pSPNS, $\triangle \mathrm{Msc}, \triangle \mathrm{MBst}$, $\Delta \mathrm{Bbs}, \Delta \mathrm{EX} \Delta \mathrm{Msc}$, and pETPLP-1 are shown. ity, including the canonical SDD polymerase motif, a nucleotide binding motif, a metal binding domain, and a helicase domain (Breedenbeek et al, 1990).

Several lines of evidence suggest that the gene 1 polyprotein is proteolytically processed into multiple polypeptides: (1) enzymatically active protease domains are encoded by ORF1a (Lee et al, 1989; Baker et al, 1989, 1993; Bonilla et al, 1995; Lu et al, 1995); (2) the extremely large size of the predicted gene 1 primary translation product, and (3) genetic evidence that as many as eight separate transacting MHV gene products are needed for RNA synthesis (Leibowitz et al, 1982; Stalcup et al, 1998). Biochemical studies using antibodies that recognize discrete portions of the protein(s) encoded by gene 1 have been useful tools to elucidate the proteolytic cleavages which process the gene 1 primary translation product (Denison et al, 1991, 1992, 1995; Hughes et al, 1995; Lu et al, 1995). To date, these studies have been most successful for ORF1a. An N-terminal $28 \mathrm{kDa}$ protein, p28, is cotranslationally cleaved from the primary translation product by the first papain-like protease encoded in ORF1a (Denison and Perlman, 1986, 1987; Baker et al, 1989; Denison et al, 1992). Subsequently, a $65 \mathrm{kDa}$ protein (p65) is cleaved from a large precursor protein and additional discrete polypeptides approximately $290 \mathrm{kDa}$ (p290), $240 \mathrm{kDa}$ (p240), $50 \mathrm{kDa}$ (p50), and p29 kDa (3CLpro) molecular mass are generated by proteases (Denison et al, 1992, 1995; Hughes et al, 1993; Piñón et al, 1997; Lu and Denison, 1997). The presumed relationships of these cleavage products to each other and their positions on the MHV genome are shown in Figure 1.

Enzymes capable of directing MHV RNA synthesis sediment with the membrane fraction of the cytosol (Brayton et al, 1982), suggesting that gene 1 encoded polypeptides might localize to a membrane bound cytoplasmic compartment. Therefore, we have studied the intracellular localization of the various protein products of ORF1a. Since the processing pathway for ORF1a gene products is much better understood than that for ORF1b, and we have in hand well characterized antibodies recognizing defined regions encoded by ORF1a, our initial efforts have been restricted to proteins encoded within this portion of the genome. We report that the majority of the p28 protein is not membrane associated but is contained in the soluble fraction of the cytosol. In contrast the downstream polypeptides p240 and p290 were localized in the Golgi region of the cell.

\section{Results}

Single color immunofluorescent labeling was performed to determine whether the various MHV ORF1a polypeptide products were differentially 
distributed in MHV-A59-infected L2 cells. Antisera UP1 recognizes the N-terminal ORF1a cleavage product p28 (Figure 1). Immunofluorescent labeling with this antiserum resulted in a diffuse somewhat granular pattern of cytoplasmic staining with focal regions labeling more heavily, indicating that this protein is widely distributed in the cytoplasm of infected cells (Figure 2A). Immunofluorescent labeling of infected cells with antisera UP102, an antisera which recognizes the p65 cleavage product as well as p28, labeled vesicular structures in the perinuclear region in addition to staining the cytoplasm more diffusely (Figure 2B). Immunofluorescent labeling of infected cells with antisera which recognize polypeptides p50, p240 and p290, antisera 81043 (Figure 2C) and 600A (not shown), gave a different pattern, intensely labeling discrete vesicular perinuclear structures in the absence of any other cytoplasmic fluorescence, suggesting that these proteins might be localized to the Golgi.

To investigate this further, two color immunofluorescent labeling was performed using a Golgispecific anti-mannosidase II monoclonal antibody (mab) in addition to the various anti-ORF1a antibodies. The mouse anti-Golgi mab 53FC3 labels murine cells poorly, if at all, but clearly labels hamster cells (Burke et al, 1982). Therefore we utilized a BHK derived cell line which expresses recombinant $\mathrm{MHV}$ receptor, $\mathrm{BHK}_{\mathrm{MHVR}}$ (Dveskler et al, 1991), for most of the subsequent experiments. $\mathrm{BHK}_{\mathrm{MHVR}}$ cells were infected with MHV-A59 or mock-infected and labeled with either the antiORF1a antisera UP1, UT2662, UP102, 81043, or $600 \mathrm{~A}$ in addition to the Golgi-specific 53FC3 mab. Figure 3A shows that most of the anti-p28 labeling (green) did not coincide with the Golgi complex which was labeled with antibody 53FC3 (red); only a modest amount of p28 antigen appeared to colocalize with the Golgi (yellow). Labeling with an anti-p65 serum gave a more complex pattern (Figure 3B). In the majority of infected cells most of the p65 labeling was not restricted to the Golgi, but was present in the peri-nuclear region with some p65 antigen colocalizing to vesicular structures which also labeled with the Golgi-specific 53FC3 mab. For approximately $25 \%$ of the cells, p65
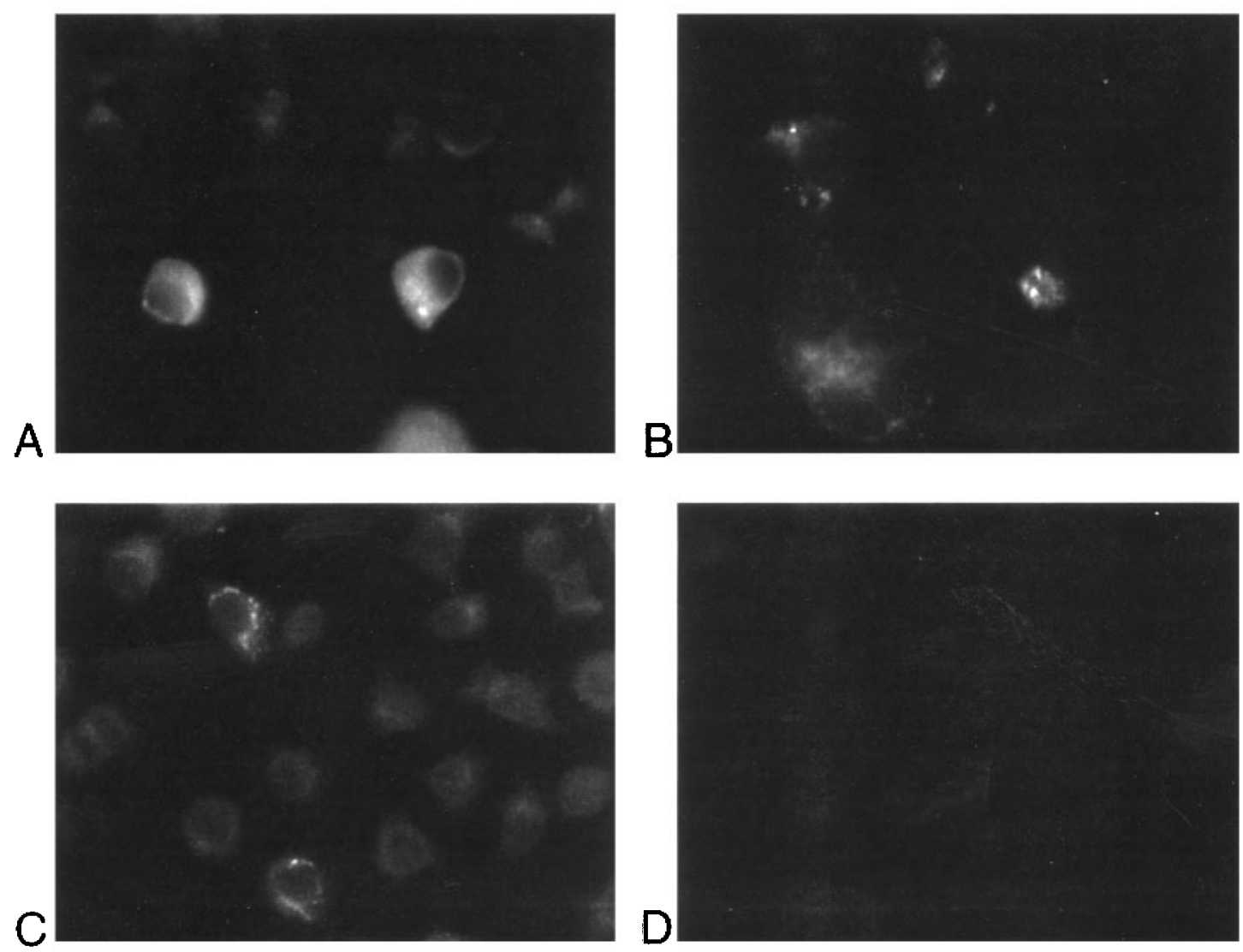

Figure 2 Immunofluorescent labeling of ORF1a products during MHV infection of mouse L2 cells. L-2 cells were infected with MHVA59 or mock-infected, incubated for $7 \mathrm{~h}$ and then fixed with acetone. Infected cells were labeled with either UP1 (A), UP102 (B), or 81043 (C) and FITC-conjugated goat anti-rabbit IgG as described in Materials and methods. Mock infected cells (D) were similarly labeled using UP1 as the primary antibody. 
antigen was confined to the Golgi region. Antiserum UP102, which recognizes both p28 and p65, labeled polypeptides which colocalized with the Golgi- specific anti-mannosidase II antibody, as well as polypeptides which appeared to have an extraGolgi location (Figure 3C). Virtually all the labeling
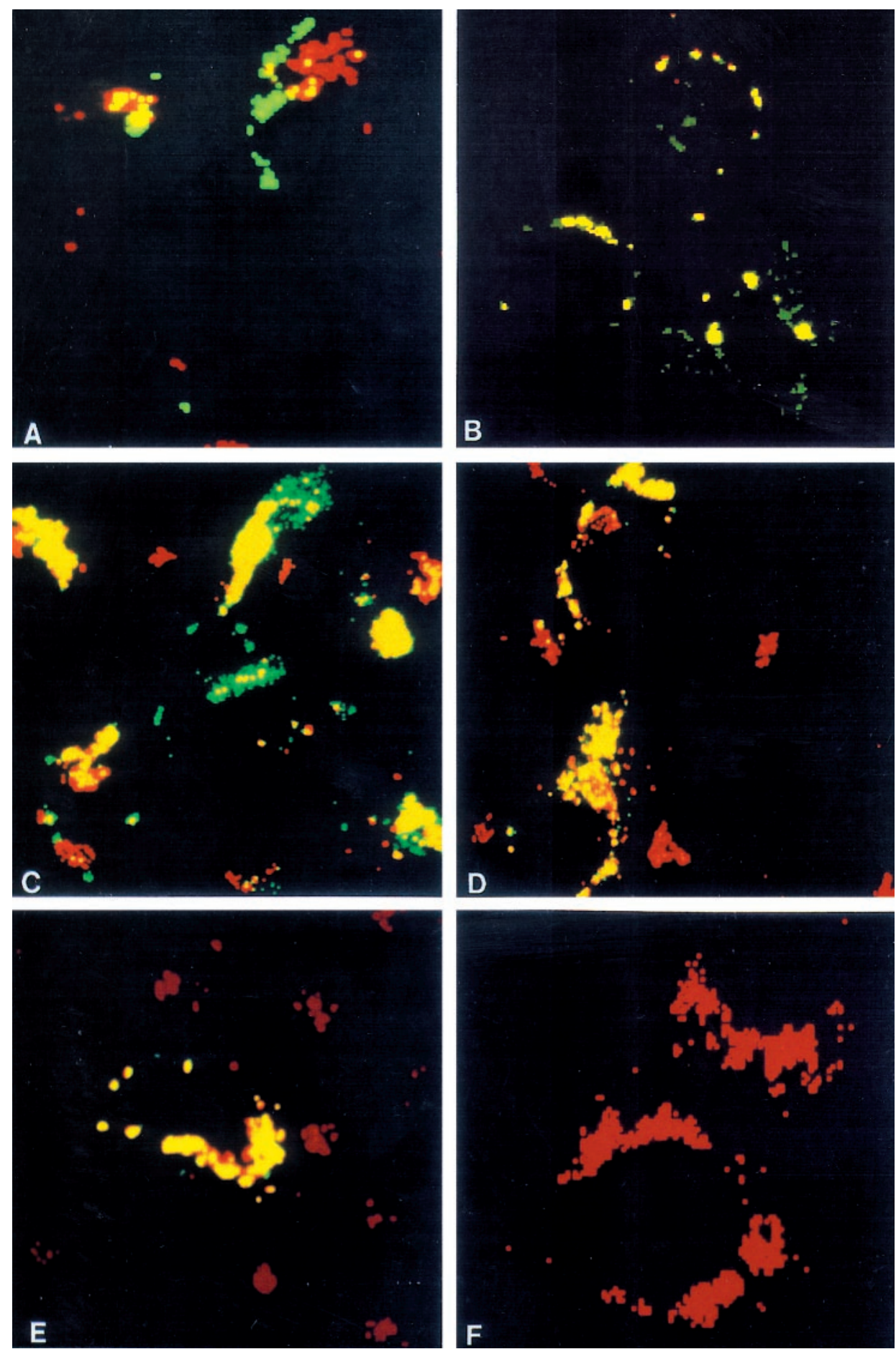

Figure 3 Immunofluorescent localization of ORF1a products in MHV-A59 infected cells. BHK cells expressing recombinant MHV receptor $\left(\mathrm{BHK}_{\mathrm{MHVR} 1}\right)$ were infected with MHV-A59 or mock-infected, incubated for $8 \mathrm{~h}$ and then fixed with acetone. Infected cells were labeled with the anti-Golgi mab 53FC3 (red) and either UP1 (A), UT2662 (B), UP102 (C), 81043 (D), or 600A (E) as described in Materials and methods. Mock infected cells (F) were stained with 53FC3 and 81043. The coverslips were viewed by confocal microscopy as described in Materials and methods. Labeling with rabbit anti-ORF1a sera (UP1, UT2662, UP102, 600A and 81043) only appears green; coincident labeling with the anti-Golgi mab and rabbit anti-ORF1a antibodies appears yellow. 
of p50 and p290 polypeptides by antiserum 81043 (Figure 3D) coincided with the labeling of the Golgi complex by mab 53FC3. Virtually no green labeling was observed, indicating that the vast majority of the polypeptide(s) recognized by 81043 is localized in the Golgi region of the cell. A similar result was obtained with antiserum 600A (Figure 3E), a serum which recognizes p50 and p290. None of the antiORF1a sera used in these experiments stained uninfected cells (Figure $3 \mathrm{~F}$ and data not shown).

Immunoprecipitation studies were done with cytoplasmic extracts which had been fractionated into soluble and membrane-containing fractions
(Figure 4). The efficiency of fractionation of the cytoplasm into soluble and membrane portions was monitored by following the partition of the MHV N (lanes 1 and 2) and M (lanes 3 and 4) proteins, which are known to be free in the cytoplasm (N) or localized to the intermediate compartment and the Golgi (M) (Holmes et al, 1981; Collins et al, 1982). The p28 protein was precipitated predominantly from the soluble cytoplasmic fraction (lane 5) rather than the membrane pellet (lane 6). Immunoprecipitation experiments with antibodies recognizing the p240 and p290 polypeptides were difficult to interpret,

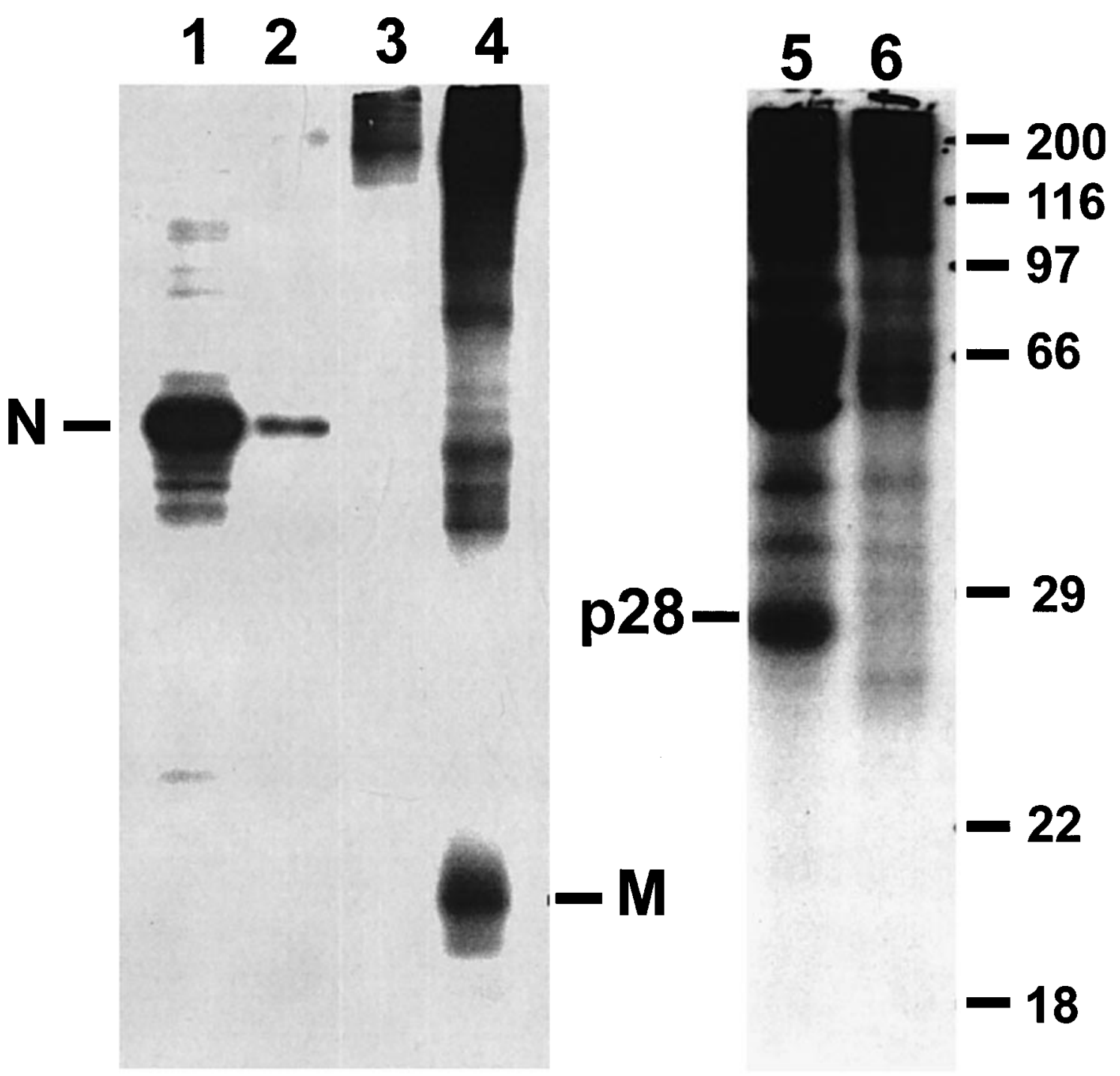

Figure 4 Subcellular fractionation of p28. MHV-infected BHK ${ }_{M H V R 1}$ cells were metabolically labeled with radioactive methionine and cysteine and cytoplasm prepared and separated into soluble and membrane-containing fractions as described in Materials and methods. Aliquots of the soluble fraction (lanes 1, 3 and 5) and the solubilized membrane-containing pellet fraction (lanes 2, 4 and 6) were immunoprecipitated with either the anti-N monoclonal antibody 1.16 .1 (lanes 1 and 2), the anti-M monoclonal antibody J2.7 (lanes 3 and 4), or with the anti-p28 antibody UP1 (lanes 5 and 6). Immunoprecipitated proteins were resolved by electrophoresis in $12 \%$ polyacrylamide gels. 
due to the precipitation of a smear of high molecular weight proteins from all samples (data not shown).

Inspection of the hydrophobicity plot of the entire predicted ORF1a polypeptide reveals that the only clearly predicted transmembrane domains are far downstream, flanking the picornavirus 3Clike protease domain. The sequences contained within p290 and p240 extend into this region of ORF1a (Figure 1). To investigate if these putative transmembrane domains are required for targeting p290 and p240 for localization in the Golgi region, we have utilized the plasmids pSPNK and pSPNS which contain the first 4482 nucleotides and 6574 nucleotides, respectively, of ORF1a under the control of the T7 promoter. These constructs do not include the two transmembrane domains surrounding the 3C-like protease domain. The polypeptides encoded by both of these constructs contain a functional papain-like protease and cleave p28 from the primary in vitro translation products (Bonilla et al, 1995; Hughes et al, 1995). These two constructs were expressed in $\mathrm{BHK}_{\mathrm{MHVR}}$ cells using the coupled vaccinia virus $\mathrm{T} 7$ polymerase system. Cells transfected with pSPNK and infected with vTF7.3 were fixed at $8.5 \mathrm{~h}$ post transfection and labeled with antisera 81043 or UP1 in combination with the anti-Golgi mab 53FC3. Since the amino acid sequences recognized by antiserum 600A are not encompassed by this plasmid, this antiserum was not used for this experiment. The labeling patterns observed were very similar to those observed during infection with MHV-A59. Labeling of p28 antigen with the UP1 antiserum demonstrated that this polypeptide did not specifically localize to the Golgi region (Figure $5 \mathrm{~A})$ but is more widespread in the cytosol. As shown in Figure 5B, all the staining of pSPNKexpressed p240/p290-related proteins by antisera 81043 coincided with the staining of the Golgi complex by antibody 53FC3. Labeling of p65 with antisera UT2662 yielded a predominantly perinuclear pattern, with most, but not all, of the labeling coincidental with labeling by the Golgi anti-mannosidase II antibody (data not shown). Additional experiments with the plasmid pSPNS produced similar results (not shown). These in vivo results showing that spacial dissociation of p28, and presumably proteolytic cleavage accompanying this spacial dissociation, from translation products which contain the first papain-like protease domain but not the second protease domain are consistent with in vitro studies showing that $\mathrm{p} 28$ cleavage only requires the first papain protease domain (Baker et al, 1989; Hughes et al, 1995).

The data present above indicated that the predicted transmembrane domains flanking the 3C-like protease domains were not necessary for localization of p290/p240 in the Golgi region of the cell. In an effort to determine the sequences targeting the polypeptide containing the epitopes recognized by antibody 81043 to the Golgi region of the cell, a series of plasmids containing internal deletions relative to pSPNK were expressed. Trans-
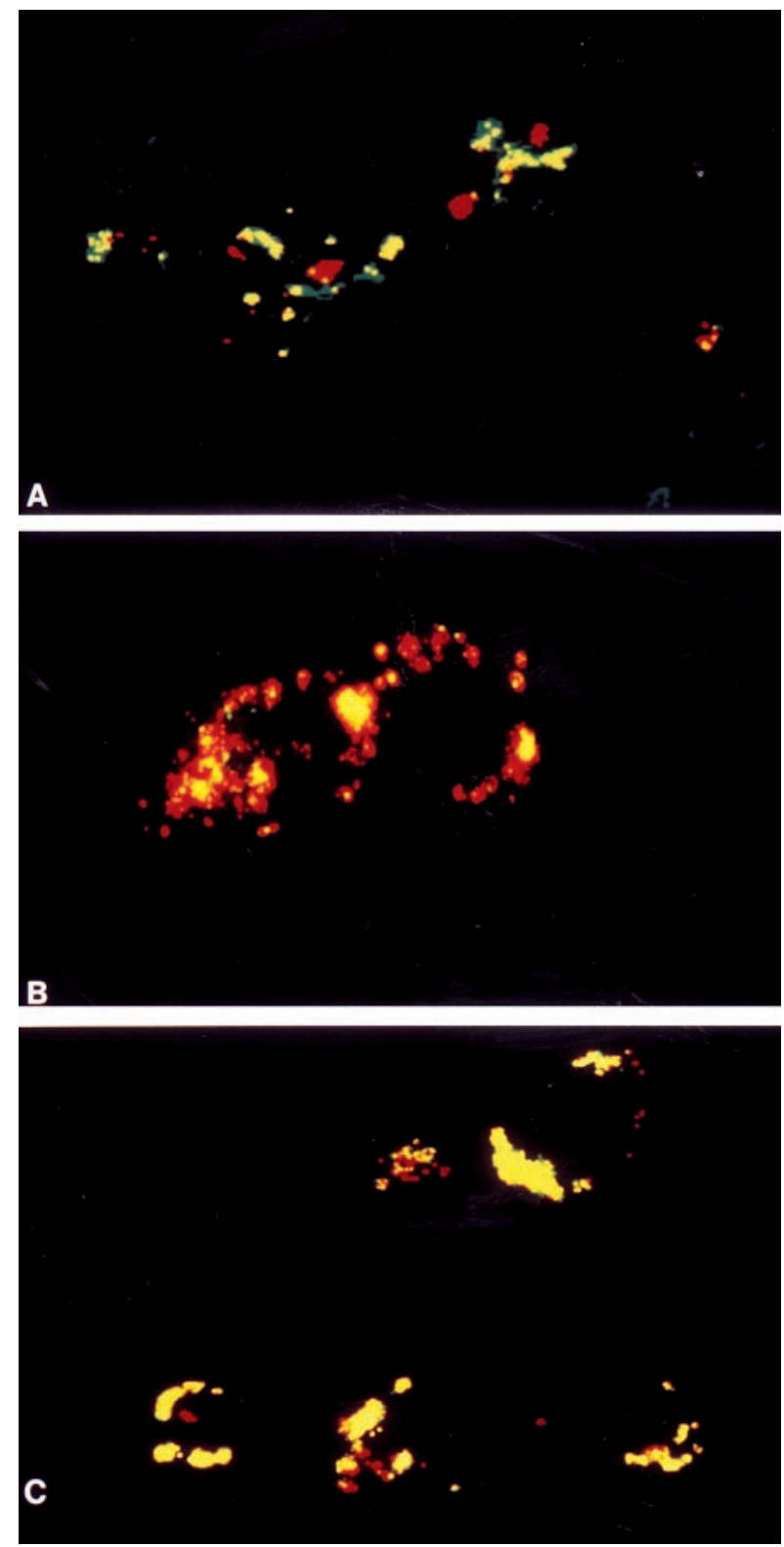

Figure 5 Immunofluorescent localization of ORF1a products in

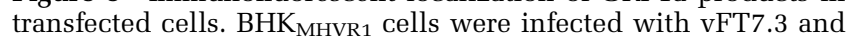
transfected with either pSPNK (A and B) or pETPLP-1 (C) as described in Materials and methods. Eight and one half hours after the addition of DNA the coverslips were fixed and subsequently labeled with the anti-Golgi mab 53FC3 (red) and with either the anti-p28 antisera UP1 (A) or antiserum 81043 (B and $\mathbf{C}$ ). The coverslips were visualized by confocal microscopy. Labeling with rabbit anti-ORF1a sera (UP1 and 81043) only appears green; coincident labeling with the anti-Golgi mab and rabbit anti-ORF1a antibodies appears yellow. 
fection of the plasmids $\Delta$ Msc, $\Delta$ MBst, $\Delta B$ bs, and $\Delta \mathrm{EX} \Delta \mathrm{Msc}$ (Bonilla et al, 1995), into vTF7.3 infected cells followed by immunofluorescent labeling with antiserum 81043 and the anti-Golgi mab 53FC3 indicated that the polypeptide segment recognized by 81043 localized to the Golgi region of the cell, in spite of the absence of various upstream polypeptide segments (data not shown). The 81043 antiserum was raised against a prokaryotic fusion protein encompassing the first papain-like domain in ORF1a. To determine if the first papain-like protease domain contained the sequences directing association with the Golgi region, the plasmid pETPLP-1 was expressed in BHK $_{\mathrm{MHVR} 1}$ cells and the location of this domain determined by double immunofluorescent labeling with antiserum 81043 and mab 53FC3. As shown in Figure 5C, the labeling pattern obtained with antiserum 81043 coincides with that obtained with 53FC3, indicating that the first papain-like protease domain encoded by pPLP1 contains sequences directing its association with the Golgi region of the cell.

During infection with MHV the bulk of the MHV M protein is retained in the pre-Golgi intermediate compartment (Tooze et al, 1984; Krijnse-Locker et al, 1994) where virus budding takes place. To investigate the likely possibility that the site of budding and the location of ORF1a proteins are different, double immunofluorescent labeling was performed using the anti-M protein monoclonal antibody (mab) J.2.7 (Fleming et al, 1989), generously provided by Dr John Fleming, and the anti-ORF1a antibody 81043. As shown in Figure 6, these two antibodies produce patterns of staining which only overlap to a limited extent. This result is consistent with our localization of the papain-like protease domain to the Golgi in Figures 3 and 5, and the accumulation of the majority of the $\mathrm{M}$ protein in the intermediate compartment.

\section{Discussion}

To better understand the functions of the MHV ORF1a encoded proteins we have initiated a series of experiments to determine their intracellular location. As reported here, p28 is more widely distributed than p290/240, as judged by immunofluorescent labeling with the UP1 antibody, and p28 partitions into the soluble fraction of the cytosol, suggesting that it is not membrane bound like the other ORF1a proteins. This was true in MHV-A59 infected L-2 and $\mathrm{BHK}_{\mathrm{MHVR}}$ cells and in $\mathrm{BHK}_{\mathrm{MHVR}}$ cells transfected with pSPNS, pSPNK, and pETPLP-1, plasmids which each contain a single papain-like protease domain (PLP1 in Figure 1). This is the first in vivo experiment which indicates that the first papain-like protease, rather than the second papainlike protease domain or the downstream 3C-like protease, cleaves p28 from the ORF1a primary translation product. Cell-free translation studies utilizing many of the same constructs employed in this work previously demonstrated that the first papain-like protease domain cleaves p28 from the ORF1a primary translation product in vitro (Bonilla et al, 1995). Thus there is good agreement between the results obtained in vivo and in vitro.
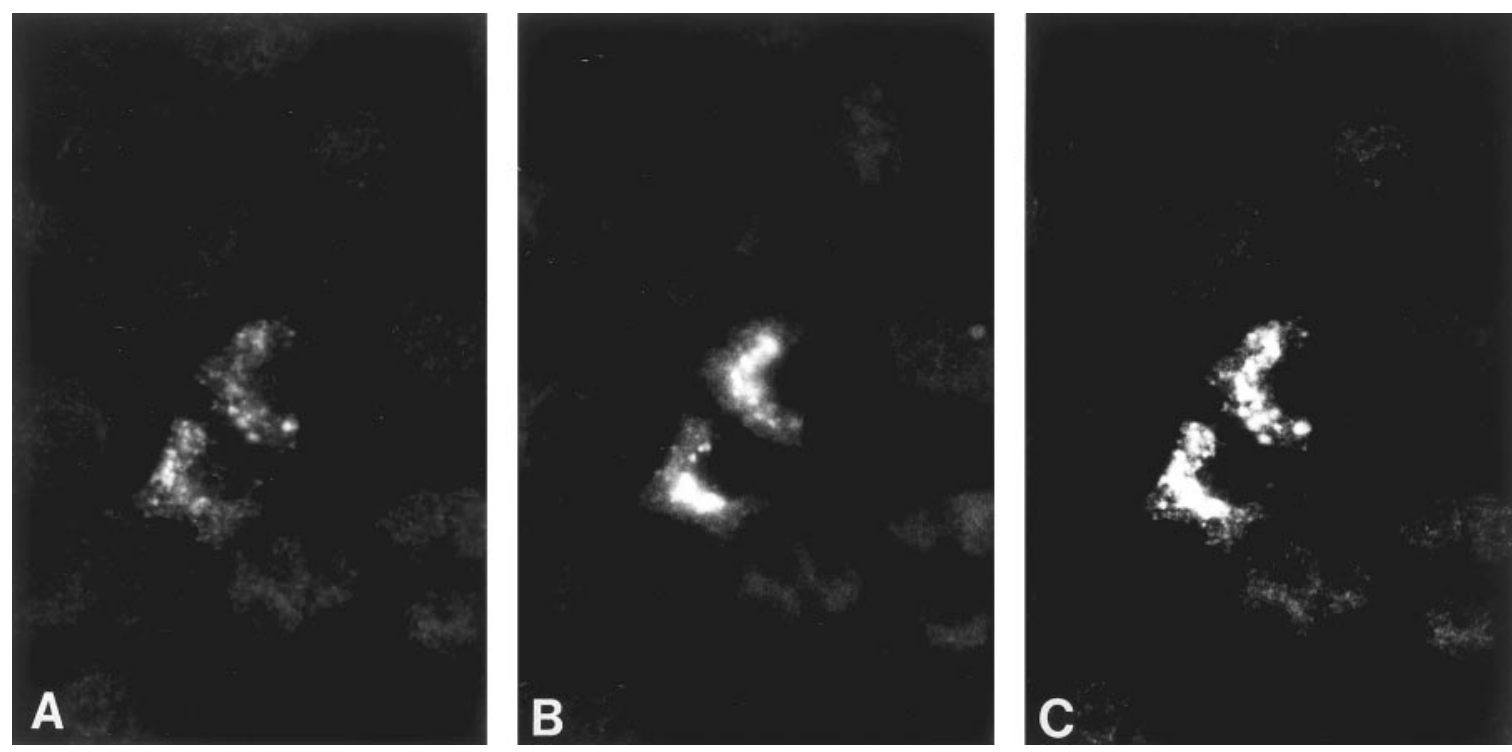

Figure 6 Double immunofluorescent labeling of ORF1a products and M protein in MHV-A59 infected cells. L2 cells were infected with MHV-A59 and fixed with acetone at $6 \mathrm{~h}$ post infection. Cells were doubly labeled with a mixture of rabbit anti-ORF1a antibody 81043 and anti-M protein monoclonal antibody J.2.7 as primary labels, and a mixture of FITC-conjugated goat anti-rabbit IgG and rhodamine-conjugated goat anti-mouse IgG as secondary labels. Antibody 81043 labeling is shown in (A). Antibody J.2.7 labeling is shown in (B). Simultaneous labeling with both antibodies is shown in (C). 
We have demonstrated by double immunofluorescent labeling that several ORF1a polypeptides colocalize to the Golgi region of the cell. Both the $600 \mathrm{~A}$ and 81043 antisera recognize the p290 and p240 ORF1a cleavage products and label the Golgi region of infected cells. The downstream putative hydrophobic transmembrane domains M1 and M2 (Figure 1) cannot be responsible for the association of p240/p290 with the Golgi, since plasmids lacking the coding sequences for these domains, pSPNK and pSPNS, directed the polypeptide product recognized by the 81043 antibody to the Golgi region of the cell. Further expression studies with the plasmid pETPLP-1 indicated that the first papain-like protease domain by itself contains sequences capable of directing the association of p240/p290 with the Golgi. Since no clear hydrophobic domain is contained within this region, it is likely that the protein is bound to Golgi membranes by protein-protein interactions. Further experiments are needed to more precisely determine the mechanism targeting these proteins to the Golgi.

The distribution of labeling for the epitopes recognized by our anti-p65 antibody was more complex. About 25\% of MHV-A59 infected cells showed a substantial degree of colocalization with the Golgi marker anti-mannosidase II. The remainder of the cells gave a less discrete perinuclear pattern of staining. We do not have a firm explanation for this cell to cell variation in the distribution of p65, although part of this variation could be due to the slower liberation of p65 from its precursor as compared to p28 (Denison et al, 1995). This precursor is thought to contain the first papain-like protease domain which we have shown associates with the Golgi when expressed in isolation, and could account for the staining pattern we observed. If this is so, than the labeling of the Golgi with UT2662 could reflect the localization of the p65 precursor polypeptide.

The differential distribution of the ORF1a proteins p28 and p240/290 allows several conclusions to be drawn. Although the function of p28 remains unknown, it is likely that the site of action for this protein is in the cytosol, distinct from the location of the PLP1-containing polypeptides which are confined to the Golgi region of the cell. Second, the localization of the p240 and p290 polypeptides containing the papain-like protease domains to the Golgi region of the cell suggests that the processing of the ORF1a translation products occurs in or near this cellular compartment. The observation that replication and transcriptionally active RNAs and RNA dependent RNA polymerase activity are associated with cell membranes (Brayton et al, 1982) raises the possibility that the polypeptides involved in MHV RNA replication and transcription could also be specifically localized to the Golgi region.
The intracellular localization of non-virion proteins thought to be required for MHV RNA synthesis to a membrane containing intracellular structure, is similar in some respects to observations made with other plus-stranded RNA viruses. Equine arteritis virus (EAV), an arterivirus, and MHV, a coronavirus, are both members of the nidovirus family, a classification based on their similar replication strategies and genetic organizations, particularly in the organization of ORF1a and ORF1b which contain genes required for viral RNA transcription and replication (den Boon et al, 1991). Recently Van Dinten et al (1996) have shown that during infection with EAV proteins containing the EAV polymerase and helicase domains are localized in the perinuclear region of the cell. They have also demonstrated an association of ORF1a derived polypeptides in a complex with these ORF1b derived proteins and have hypothesized the formation of a membrane bound replicase complex consisting of ORF1a and ORF1b derived proteins. Our data is not completely consistent with extending this concept to the coronaviruses. We have demonstrated that MHV ORF1a PLP1 containing polypeptides colocalize with the Golgi, an observation which is consistent with the underlying similarity of the arterivirus and coronavirus systems and the homology of the PLP1 domain encoded by the two virus groups. However, the MHV p28 and p65 polypeptides are not confined to the Golgi and are inconsistent with a model in which all of the ORF1a and ORF1b proteins are located in a replicase complex. There are no obvious homologs to the MHV p28 and p65 polypeptides in the arterivirus genome, thus our results for these proteins may not apply to the arteriviruses.

Cells infected with either poliovirus or Sindbis contain de novo synthesized vesicular structures containing polypeptides which are components of the viral RNA biosynthetic machinery (Bienz et al, 1992; Miller and Brown, 1993). For poliovirus it is clear that these structures are sites of RNA replication (Takeda et al, 1986; Bienz et al, 1992). In this respect our results differ somewhat from those obtained with these two viruses, in that the MHV proteins we have studied localized to a preexisting structure, namely the Golgi complex. However, we have not yet demonstrated the intracellular location of MHV replication complexes. During infection with MHV the assembly of viral nucleocapsids and virus budding takes place primarily in smooth-walled vesicles transitional between the Golgi and ER (Tooze et al, 1984), and it has been suggested that RNA synthesis might be localized to this compartment (Dubois-Dalcq et al, 1984). Future studies with antibodies which recognize downstream ORF1b domains predicted to be components of the MHV RNA replication and transcription machinery 
coupled with in vitro biochemical studies of MHV transcription are needed to better address these issues.

\section{Materials and methods}

Cells and virus

The origin and growth of L-2 cells and BHK cells $\left(\mathrm{BHK}_{\mathrm{MHVR}}\right)$ expressing recombinant $\mathrm{MHV}$ receptor have been described (Leibowitz et al, 1981; Dveksler et al, 1991). The origin and growth of MHV-A59 has been described (Leibowitz et al, 1981). The recombinant vaccinia virus expressing the bacteriophage T7 RNA polymerase, vTF7.3 was kindly provided by Dr Bernard Moss (Fuerst et al, 1987).

\section{Antibodies}

Monoclonal antibodies J2.7 and 1.16.1 which recognize the membrane $(\mathrm{M})$ protein and the nucleocapsid $(\mathrm{N})$ protein, respectively, have been described previously (Fleming et al, 1989; Denison et al, 1991).

Most of the polyclonal antibodies used in this study (UP1, UP102, 600A, 81043) have been described previously (Denison et al, 1992, 1995; Hughes et al, 1993). These antisera were raised in rabbits using prokaryotic/viral fusion proteins as immunogens. An antibody recognizing p65, the ORF1a cleavage product immediately downstream from the N-terminal p28 cleavage product, was raised by immunizing a goat with a purified polyhistidine-tagged polypeptide expressed in $E$. coli. A cDNA fragment encoding a portion of the p65 polypeptide (nucleotides 1376-1774, Bonilla et al, 1994; Denison et al, 1995) was amplified by PCR using an upstream primer, CCGGATCCTTGTTGTGGTGACACCTGCG, which introduced a BamHI site onto the $5^{\prime}$ end of the fragment, and a downstream primer, AACAAGTGGCTTCGCTGC. The amplified cDNA fragment was digested with XhoI, filled in with Klenow, digested with BamHI and the resulting 399 nucleotide fragment cloned into the plasmid pET15b (Novagen) utilizing a BamHI site and a Bpu1 102I site which had been filled in with Klenow (Rosenberg et al, 1987; Studier et al, 1990). The resulting plasmid, pET15b-p65, contained 132 amino acids of p65 fused to an upstream polyhistidine sequence via a vectorderived synthetic sequence containing a thrombin cleavage site, all under the control of the T7 promoter. The plasmid carrying this construct was transferred to the inducible T7 polymerase expressing strain, BL21(DE3). The histidine-tagged polypeptide was induced with $1 \mathrm{mM}$ IPTG and partially purified by affinity chromatography over a nickelcontaining resin using the directions supplied by the vendor (Novagen). Further purification of the histidine-tagged p65 fragment was achieved by preparative SDS-PAGE. Antibodies to p65 were prepared by Bethyl Laboratories, Inc. (Montgomery, TX, USA). A goat was immunized once subcutaneously with $100 \mu \mathrm{g}$ of purified protein emulsified in complete Freund's adjuvant and boosted six times at biweekly intervals with the same amount of antigen in incomplete Freund's adjuvant. The resulting antiserum was designated UT2662. The reactivity of UT2662 with p65 was verified by immunoprecipitation of coupled in vitro transcription/translation reactions programmed with pET15b-p65 as template.

The locations of the viral sequences encoding the various polypeptides used to raise these antibodies, as well as the locations of the proteins recognized by these antibodies, are summarized in Table 1 and depicted schematically in Figure 1. The Golgispecific anti-mannosidase II monoclonal antibody 53FC3 was purchased from the Berkeley Antibody Company (Burke et al, 1982). This antibody labels hamster but not mouse Golgi complex. Fluoresceinconjugated goat anti-rabbit IgG and rhodamineconjugated goat anti-mouse IgG were purchased from Jackson Research.

\section{Plasmids and transfections}

Plasmids pSPNK and pSPNS are derived from cDNAs and contain MHV-A59 gene 1 sequences from the NarI site at nucleotide 182 to the KpnI site at nucleotide 4664 and the SpeI site at nucleotide 6756, respectively (Bonilla et al, 1994; 1995; 1997). In both cases the MHV-A59 cDNA is oriented in the plasmid pSP72 such that the coding sequences are under the control of the T7 promoter. pSPNK contains the coding sequence for $\mathrm{p} 28$, p65, and the first papain-like domain of MHV-A59; pSPNS extends past the second papain-like domain (Figure

Table 1 Antisera used for immunofluorescent localization of A59 ORF1a products.

\begin{tabular}{lccc}
\hline Antisera & $\begin{array}{c}\text { Size of viral sequences used as } \\
\text { immunogen (aa) }\end{array}$ & $\begin{array}{c}\text { cDNA for fusion proteins spans } \\
\text { nucleotides }\end{array}$ & $\begin{array}{c}\text { Polypeptides immunoprecipitated } \\
\text { from infected cells }\end{array}$ \\
\hline UP1 & 228 & $461-1144$ & $\mathrm{p} 28$ \\
UP102 & 601 & $182-1984$ & $\mathrm{p} 28, \mathrm{p} 65$ \\
UT2662 & 132 & $1376-1774$ & $\mathrm{p} 65$ \\
81043 & 363 & $2879-3968$ & $\mathrm{p} 290, \mathrm{p} 240$ \\
600A & 495 & $5000-6486$ & $\mathrm{p} 290, \mathrm{p} 240$ \\
\hline
\end{tabular}


1). The plasmids $\Delta \mathrm{Msc}, \Delta \mathrm{MBst}, \Delta \mathrm{Bbs}$, and $\Delta \mathrm{EX} \Delta \mathrm{Msc}$ are derivatives of pSPNK containing a series of in-frame deletions between the p28 cleavage site and the first papain-like domain (Bonilla et al, 1995). The plasmid pETPLP-1 contains ORF1a sequences from nucleotides $3393-4301$. This sequence was amplified by PCR using primers which introduced an NdeI site upstream of the ORF1a sequence and an XbaI site downstream. The NdeI site provides an initiation codon for protein synthesis. The amplified DNA was then cloned into the NdeI and XbaI sites of the vector pHB40P. PHB40P is a derivative of pET3a and contains $K p n I, X b a \mathrm{I}$, and $X h o I$ sites inserted between the BgIII and BamHI sites in the parent vector (Rosenberg et al, 1987; Studier et al, 1990).

For transfection experiments, $60 \mathrm{~mm}$ dishes containing sterile coverslips were seeded with $2.2 \times 10^{6} \mathrm{BHK}_{\mathrm{MHVR}}$ cells and incubated overnight. BHK $_{\mathrm{MHVR}}$ cells were used rather than murine cells to allow subsequent immuno-labeling with the Golgispecific 53FC3 monoclonal antibody. The cells were then infected at a MOI=5 with recombinant vaccinia virus expressing T7 polymerase, vTF7.3. The cultures were incubated at $37^{\circ} \mathrm{C}$ for $2.5 \mathrm{~h}$ and then transfected with $5 \mu \mathrm{g}$ of plasmid using lipofectin (Life Technologies) according to the manufacturer's instructions. After further incubation for $2.5 \mathrm{~h}$ the transfection medium was replaced with fresh DME, and the cells were incubated for a further $6 \mathrm{~h}$, at which time they were washed with PBS and fixed with cold acetone.

\section{Indirect immunofluorescence}

L-2 cells and $\mathrm{BHK}_{\mathrm{MHVR}}$ cells were grown for 2 days on glass coverslips or chamber slides and infected with MHV-A59 at a calculated MOI of 0.3 or mock-infected. At 6-7 h post-infection (L-2 cells), or at $8 \mathrm{~h}$ post-infection (BHK $\mathrm{MHVR}_{\mathrm{M}}$ cells), cells were fixed for 5 min with cold acetone. For transfection experiments, $\mathrm{BHK}_{\mathrm{MHVR}}$ cells were similarly fixed $8.5 \mathrm{~h}$ after transfection. The fixed coverslips were washed twice with phosphate buffered saline, pH 7.4 (PBS) containing $0.2 \%$ Tween 20 and treated with $10 \%$ non-fat milk in PBS overnight at $4{ }^{\circ} \mathrm{C}$ to block non-specific staining. The coverslips were then washed 2-3 times with PBS containing $0.2 \%$ Tween 20 , and subsequently incubated on ice with rabbit anti-ORF1a antibody (1:100 dilution in PBS containing 3\% BSA) for 90 min. Following incubation with primary antibody, the coverslips were washed extensively with PBS containing $0.2 \%$ Tween 20, incubated for 60 min with FITC-conjugated goat anti-rabbit IgG (1:100 dilution), and washed five times with PBS containing $0.2 \%$ Tween 20. Coverslips were mounted and examined by fluorescent microscopy. For double labeling experiments, a mixture of rabbit anti-ORF1a antibodies (diluted 1:100) and mouse monoclonal anti-mannosidase II anti- body (diluted 1:600) was used as a primary label, and a mixture of FITC-conjugated goat anti-rabbit IgG and rhodamine-conjugated goat anti-mouse IgG was used in a secondary labeling step. The cells were mounted in elvanol mounting solution for examination with a Multi-probe 2001, CLSM confocal microscope (Molecular Dynamics). The CLSM produces simultaneous dual channel images. Colocalization of the two chromophores was revealed by the yellow color resulting from their overlapping emission.

\section{Immunoprecipitation and cell fractionation}

Confluent monolayers of L2 cells in $35 \mathrm{~mm}$ wells were infected with A59 at an MOI of 10. The infected monolayers were incubated with DME containing one tenth of the normal amount of methionine and cysteine and supplemented with $2 \%$ FBS. At $2 \mathrm{~h}$ post infection actinomycin $\mathrm{D}$ was added to the medium to a concentration of $10 \mu \mathrm{g} / \mathrm{ml}$. At $7 \mathrm{~h}$ post infection, a time when approximately $50 \%$ of the cells were fused to form large syncytia, monolayers were washed with methionine-free and cysteine-free DME, and labeled with $250 \mu \mathrm{Ci}$ of $\left[{ }^{35} \mathrm{~S}\right]$-methionine and $\left[{ }^{35} \mathrm{~S}\right]$-cysteine in $0.5 \mathrm{ml}$ of the same medium for $2 \mathrm{~h}$. Monolayers were harvested and fractionated essentially as described previously (Zoltick et al, 1990). After labeling, the monolayers were washed five times with buffer A $(10 \mathrm{mM}$ Tris, pH 7.4, $150 \mathrm{mM} \mathrm{NaCl}, \quad 80 \mu \mathrm{g} / \mathrm{ml}$ PMSF), scraped into $1 \mathrm{ml}$ of the same buffer, and collected by centrifugation for $5 \mathrm{~min}$ at $500 \mathrm{~g}$. The cells were resuspended in $0.3 \mathrm{M}$ sucrose, held on ice for $10 \mathrm{~min}$, and disrupted with 15 strokes of a Dounce homogenizer. As judged by phase microscopy, more than $90 \%$ of the cells were broken by this procedure. Nuclei and unbroken cells were removed by centrifugation at $800 \mathrm{~g}$ for $8 \mathrm{~min}$. The crude post-nuclear extract was fractionated into soluble and membrane-containing fractions by centrifugation at $13000 \mathrm{~g}$ for $20 \mathrm{~min}$. The membrane-containing pellet was solubilized in $100 \mu \mathrm{l}$ of Buffer B (10 mM Tris, pH 7.4; $150 \mathrm{mM} \mathrm{NaCl,} \mathrm{1 \%} \mathrm{NP-40,}$ $1 \%$ sodium deoxycholate, $0.1 \%$ SDS). An equal volume of $2 \times$ Buffer $B$ was added to the soluble fraction of the post-nuclear supernate.

Soluble and membrane-containing samples representing approximately equal numbers of cells $(25 \%$ of a $35 \mathrm{~mm}$ well) were used for each immunoprecipitation reaction. Immunoprecipitation was performed as described previously ( $\mathrm{Yu}$ et al, 1994). When monoclonal antibodies were used for immunoprecipitation, Protein A beads were precoated with goat anti-mouse IgG. Immunoprecipitates were resolved by SDSPAGE on $12 \%$ polyacrylamide gels and the immunoprecipitated proteins detected by fluorography. 


\section{Acknowledgements}

This work was supported in part by National Multiple Sclerosis Society Research Grants RG 2203-A-5 and RG 2203-B-6, and NIH grants AI17418 and AI25231. We would like to acknowl- edge the technical assistance of Elena Belyavskya and help of our student workers, Russell Canham, Paul Kurian, and Jimmy Pavliska. A special thanks to Dr Rajesh Miranda for the extensive use of his fluorescent microscope.

\section{References}

Baker SC, Shieh C-K, Soe LH, Chang M-F, Vannier DM, Lai MMC (1989). Identification of a domain required for autoproteolytic cleavage of murine coronavirus gene A polyprotein. J Virol 63: 3693-3699.

Baker SC, Yokomori K, Dong S, Carlisle R, Gorbalenya AE, Koonin EV, Lai MMC (1993). Identification of the catalytic sites of a papain-like cysteine proteinase of murine coronavirus. J Virol 67: 6056-6063.

Bienz K, Egger D, Pfister T, Troxler M (1992). Structural and functional characterization of poliovirus replication complex. J Virol 66: 2740-2747.

Bonilla PJ, Gorbalenya AE, Weiss SR (1994). Mouse hepatitis virus strain A59 RNA polymerase gene ORF1a, Heterogeneity among MHV strains. Virology 198: $736-740$.

Bonilla PJ, Hughes SA, Piñón JD, Weiss SR (1995). Characterization of the leader papain-like proteinase of MHV-A59, identification of a new in vitro cleavage site. Virology 209: 489-497.

Bonilla PJ, Hughes SA, Weiss SR (1997). Characterization of a second cleavage site and demonstration of activity in trans by the papain-like proteinase of the murine coronavirus mouse hepatitis virus strain A59. J Virol 71: 900-909.

Brayton PR, Lai MMC, Patton CD, Stohlman SA (1982). Characterization of two RNA polymerase activities induced by mouse hepatitis virus. J Virol 42: $847-$ 853.

Breedenbeek PJ, Pachuk CJ, Noten AFH, Charité J, Luyjtes W, Weiss SR, Spaan WJM (1990). The primary structure and expression of the second open reading frame of the polymerase gene of the coronavirus MHV-A59; a highly conserved polymerase is expressed by an efficient ribosomal frameshifting mechanism. Nucleic Acids Res 18: 1825-1832.

Burke B, Griffiths G, Reggio H, Louvard D, Warren G (1982). A monoclonal antibody against a 135-K Golgi membrane protein. EMBO J 1: 1621-1628.

Burks JS, DeVald BL, Jankovsky LD, Gerdes JC (1980). Two coronaviruses isolated from central nervous tissue of two multiple sclerosis patients. Science 209: $933-934$.

Collins AR, Knobler RL, Powell H, Buchmeier MJ (1982). Monoclonal antibodies to murine hepatitis virus-4 (strain JHM) define the viral glycoprotein responsible for attachment and cell-cell fusion. Virology 119: $58-$ 71.

den Boon JA, Snijder EJ, Chirnside ED, de Vries AA, Horzinek MC, Spaan WJ (1991). Equine arteritis virus is not a togavirus but belongs to the coronaviruslike superfamily. J Virol 65: 2910-2920.

Denison MR, Perlman S (1986). Translation and processing of mouse hepatitis virus virion RNA in a cell-free system. J Virol 60: 12-18.

Denison MR, Perlman S (1987). Identification of a putative polymerase gene product in cells infected with murine coronavirus A59. Virology 157: 565-568.

Denison MR, Zoltick PW, Leibowitz JL, Pachuk CJ, Weiss SR (1991). Identification of polypeptides encoded in ORF1b of the putative polymerase gene of the murine coronavirus MHV-A59. J Virol 65: 3076-3082.

Denison MR, Zoltick PW, Hughes SA, Giangreco B, Olson AL, Perlman S, Leibowitz JL, Weiss SR (1992). Intracellular processing of the N-terminal ORF1a proteins of the coronavirus MHV-A59 requires multiple proteolytic events. Virology 189: $274-284$.

Denison MR, Hughes SA, Weiss SR (1995). Identification and characterization of a $65-\mathrm{kDa}$ protein processed from the gene 1 of the murine coronavirus MHV-A59. Virology 207: $316-320$.

Dubois-Dalcq M, Holmes KV, Rentier P (1984). Assembly of RNA Viruses, pp. 100-119. Springer-Verlag, Wien, Austria and New York, NY.

Dveksler GS, Pensiero MN, Cardelluchio CB, Williams RK, Jiang GS, Holmes KV, Dieffenbach CW (1991). Cloning of the mouse hepatitis virus (MHV) receptor, expression in human and hamster cell lines confers susceptibility to MHV. J Virol 65: 6881-6891.

Fleming JO, Shubin RA, Sussman MA, Casteel N, Stohlman SA (1989). Monoclonal antibodies to the matrix (E1) glycoprotein of mouse hepatitis virus protect mice from encephalitis. Virology 168: 162167.

Fuerst T, Earl P, Moss B (1987). Use of a hybrid vaccinia virus-T7 RNA polymerase system for expression of target genes. Mol Cell Biol 7: 2538-2544.

Holmes KV, Doller EW, Behnke JN (1981). Analysis of the functions of coronavirus glycoproteins by differential inhibition of synthesis with tunicamycin. $A d v$ Exp Med Biol 142: 133-142.

Hughes SA, Bonilla P, Denison MR, Leibowitz JL, Baric RS, Weiss SR (1993). A newly identified MHV-A59 ORF1a polypeptide p65 is temperature sensitive in two RNA negative mutants. Adv Exp Med Biol 342: $221-226$.

Hughes SA, Bonilla PJ, Weiss SR (1995). Identification of the murine coronavirus p28 cleavage site. J Virol 69: $809-813$.

Krijnse-Locker J, Ericsson M, Rottier PJ, Griffiths G (1994). Characterization of the budding compartment of mouse hepatitis virus: evidence that transport from the RER to the Golgi complex requires only one vesicular transport step. J Cell Biol 124: 55-70.

Lavi E, Gilden DH, Wrobleska Z, Rorke LB, Weiss SR (1984). Experimental demyelination produced by the A59 strain of mouse hepatitis virus. Neurology 34: $597-603$. 
Lee H-J, Shieh C-K, Gorbalenya AE, Koonin EV, LaMonica N, Tuler J, Bagdzhadzhyan A, Lai MMC (1989). The complete sequence (22 kilobases) of murine coronavirus gene-1 encoding the putative proteases and RNA polymerase. Virology 180: 567582.

Leibowitz JL, Wilhelmsen KC, Bond CW (1981). The virus-specific intracellular RNA species of two murine coronaviruses, MHV-A59 and MHV-JHM. Virology 114: $39-51$.

Leibowitz JL, DeVries JR, Haspel MV (1982). Genetic analysis of murine hepatitis virus strain JHM. J Virol 42: $1080-1087$.

Lu Y, Lu X, Denison MR (1995). Identification and characterization of a serine-like proteinase of the murine coronavirus MHV-A59. J Virol 69: 3554-3559.

$\mathrm{Lu} \mathrm{Y,} \mathrm{Denison} \mathrm{MR} \mathrm{(1997).} \mathrm{Determinants} \mathrm{of} \mathrm{muse}$ hepatitis virus 3C-like proteinase activity. Virology 230: $335-342$.

Miller ML, Brown DT (1993). The distribution of Sinbis virus proteins in mosquito cells as determined by immunofluorescence and immunoelectron microscopy. J Gen Virol 74: 293-298.

Murray RS, Brown B, Brian D, Cabirac GF (1992). Detection of coronavirus RNA and antigen in multiple sclerosis brain. Ann Neurol 31: 525-533.

Piñón JD, Mayreddy RR, Turner JD, Khan FS, Bonnila PJ, Weiss SR (1997). Efficient autoproteolytic processing of the MHV-A59 3C-like proteinase from the flanking hydrophobic domains requires membranes. Virology 230: $309-322$.

Rosenberg AH, Lade BN, Chui D-S, Lin S-W, Dunn JJ, Studier FW (1987). Vectors for selective expression of cloned DNAs by T7 RNA polymerase. Gene 56: 125135.

Siddell SG, Wege H, ter Meulen V (1983). The biology of coronaviruses. J Gen Virol 64: 761-77.

Spaan WJ, Cavanagh D, Horzinek MC (1988). Coronaviruses, structure and genome expression. J Gen Virol 69: $2939-2952$.
Stalcup RP, Baric RS, Leibowitz JL (1998). Genetic complementation amongst three panels of mouse hepatitis virus gene 1 mutants. Virology 241: 112121.

Studier FW, Rosenberg AH, Dunn JJ, Dubendorff JW (1990). Use of T7 RNA polymerase to direct expression of cloned genes. Methods in Enzymology 185: $60-89$.

Talbot PJ, Ekande S, Cashman NR, Mounir S, Stewart JN (1993). Neurotropism of human coronavirus 229E. Adv Exp Med Biol 342: 339-346.

Tanaka R, Iwasaki Y, Koprowski H (1976). Ultrastructural studies of perivascular cuffing cells in multiple sclerosis brain. J Neurol Sci 28: 121-126.

Takeda N, Kuhn RJ, Yang CF, Takegami T, Wimmer E (1986). Initiation of poliovirus plus-strand RNA synthesis in a membrane complex of infected HeLa cells. J. Virol. 60: $43-53$.

Tooze J, Tooze S, Warren G (1984). Replication of coronavirus MHV-A59 in sac-cells: determination of the first site of budding of progeny virions. Eur J Cell Biol 33: 281-293.

van Dinten LC, Wassenaar AL, Gorbalenya AE, Spaan WJ, Snijder EJ (1996). Processing of the equine arteritis virus replicase ORF1b protein: identification of cleavage products containing the putative viral polymerase and helicase domains. J Virol 70: 6625-6633.

Weiner LP (1973). Pathogenesis of demyelination induced by a mouse hepatitis virus (JHM virus). Arch. Neurol. 28: 298-303.

Yu X, Bi W, Weiss SR, Leibowitz JL (1994). Mouse hepatitis gene $5 \mathrm{~b}$ protein is a new virion envelope protein. Virology 202: 1018-1023.

Zoltick PW, Leibowitz JL, Oleszak EL, Weiss SR (1990). Mouse hepatitis virus ORF2a is expressed in the cytosol of infected mouse fibroblasts. Virology 174: $605-607$ 